\title{
A geometric approach to separate the effects of magnetic susceptibility and chemical shift/exchange in a phantom with isotropic magnetic susceptibility
}

Hyunsung Eun ${ }^{1 *}$, Hwihun Jeong ${ }^{1 *}$, Jingu Lee ${ }^{1}$, Hyeong-geol Shin ${ }^{1}$, and Jongho Lee ${ }^{1}$

* Hyunsung Eun and Hwihun Jeong contributed equally to this work.

\begin{abstract}
Author affiliations:
${ }^{1}$ Laboratory for Imaging Science and Technology, Department of Electrical and Computer Engineering, Seoul National University, Seoul, Korea
\end{abstract}

\section{Correspondence}

Jongho Lee, Ph. D

Department of Electrical and Computer Engineering, Seoul National University

Building 301, Room 1008, 1 Gwanak-ro, Gwanak-gu, Seoul, Korea

Tel: 82-2-880-7310

E-mail: jonghoyi@snu.ac.kr

Word count: 3,153 


\begin{abstract}
Purpose: To separate the effects of magnetic susceptibility and chemical shift/exchange in a phantom with isotropic magnetic susceptibility. To generate a chemical shift/exchange-corrected quantitative susceptibility mapping (QSM) result.

Theory and Methods: Magnetic susceptibility and chemical shift/exchange are the properties of a material. Both are known to induce the resonance frequency shift in MRI. In current QSM, the susceptibility is reconstructed from the frequency shift, ignoring the contribution of the chemical shift/exchange. In this work, a simple geometric approach, which averages the frequency shift maps from three orthogonal $\mathrm{B}_{0}$ directions to generate a chemical shift/exchange map, is developed using the fact that the average nullifies the (isotropic) susceptibility effects. The resulting chemical shift/exchange map is subtracted from the total frequency shift, producing a frequency shift map solely from susceptibility. Finally, this frequency shift map is reconstructed to a susceptibility map using a QSM algorithm. The proposed method is validated in numerical simulations and applied to phantom experiments with olive oil, bovine serum albumin, ferritin, and iron oxide solutions.
\end{abstract}

Results: Both simulations and experiments confirm that the method successfully separates the contributions of the susceptibility and chemical shift/exchange, reporting the susceptibility and chemical shift/exchange of olive oil (susceptibility: $0.62 \mathrm{ppm}$, chemical shift: $-3.60 \mathrm{ppm}$ ), bovine serum albumin (susceptibility: $-0.059 \mathrm{ppm}$, chemical shift: $0.008 \mathrm{ppm}$ ), ferritin (susceptibility: 0.125 ppm, chemical shift: $-0.005 \mathrm{ppm}$ ), and iron oxide (susceptibility: $0.30 \mathrm{ppm}$, chemical shift: $-0.039 \mathrm{ppm})$ solutions.

Conclusion: The proposed method successfully separates the susceptibility and chemical shift/exchange in phantoms with isotropic magnetic susceptibility.

\title{
KEYWORDS
}

Quantitative MR, Magnetic susceptibility, Chemical exchange, Chemical shift, Quantitative susceptibility mapping (QSM) 


\section{INTRODUCTION}

With the development of ultra-high field MRI systems, phase shift or resonance frequency shift has become a vital contrast for high-resolution anatomy. ${ }^{1-3}$ The origins of the resonance frequency shift have been attributed to the effect of magnetic susceptibility, ${ }^{4-6}$ chemical shift, ${ }^{7}$ chemical exchange, ${ }^{8-11}$ and complex tissue microstructure. ${ }^{12-14}$ Among them, the magnetic susceptibility effect has been suggested as a primary contributor to the resonance frequency shift. Under this assumption, quantitative susceptibility mapping (QSM) has been proposed by converting a frequency shift image to a susceptibility map. ${ }^{15-19}$ The method has been demonstrated to generate great details of anatomical structures and has been applied for various clinical studies. ${ }^{20-24}$ However, the accuracy of QSM degrades when other sources of frequency shift exist. For example, in abdominal QSM, the chemical shift from fat introduces substantial artifacts in QSM images if it is reconstructed without consideration of the chemical shift of fat. ${ }^{25,26}$ Efforts were made to design models that separated the susceptibility and non-susceptibility sources but they required specific conditions to hold (e.g. a piece-wise constant assumption). ${ }^{27,28}$

The magnetic susceptibility and chemical shift/exchange are the properties of a material (e.g., vegetable oil has the magnetic susceptibility of $0.65 \mathrm{ppm}$ and chemical shift of $-3.46 \mathrm{ppm} ;{ }^{28}$ bovine serum albumin solution $(100 \mathrm{mg} / \mathrm{ml})$ has the magnetic susceptibility of $-0.068 \mathrm{ppm}$ and chemical exchange of $0.008 \mathrm{ppm}^{29}$; see Discussion). Hence, understanding the contribution of the two sources is important not only for the reliable reconstruction of QSM, but also for the separate measurements of the two sources. In a few previous studies, ${ }^{8-10}$ efforts have been made to separate the effects of the magnetic susceptibility and chemical exchange by mixing a material of interest with a reference chemical (e.g., dioxane). However, a more recent study has suggested a nonnegligible level of interactions between the reference chemical and the material under investigation. ${ }^{30}$ As a result, further research is necessary to determine the validity of the reference chemical-based measurements.

In this study, we present a novel geometric method that separates the susceptibility and chemical shift/exchange without requiring a reference chemical that is mixed with the sample of interest. A mathematical formation that utilizes multiple $\mathrm{B}_{0}$ orientation data has been developed to achieve the separation. Numerical simulation and phantom experiments were performed to validate the method. 


\section{THEORY}

\subsection{Resonance frequency shift}

When the effect of the susceptibility and chemical shift/exchange coexist in an isotropic medium (i.e. no anisotropic susceptibility, no anisotropic microstructure, and no orientationdependent chemical shift/exchange) with no additional sources for the frequency shift, the total frequency shift can be written as

$$
\Delta f(\vec{r})=f_{c}(\vec{r})+f_{s}(\vec{r}),
$$

where $\Delta f$ is the total resonance frequency shift, $\vec{r}$ is a position vector, $f_{c}$ is the chemical shift/exchange-induced frequency shift, and $f_{s}$ is the susceptibility-induced frequency shift. The susceptibility-induced frequency shift has been shown to be modeled as: ${ }^{31,32}$

$$
\begin{gathered}
f_{S}(\vec{r})=d * \chi(\vec{r}), \\
d(\vec{r})=\frac{1}{4 \pi} \cdot \frac{3 \cos ^{2} \theta-1}{\vec{r}^{3}},
\end{gathered}
$$

where $d$ is a dipole kernel, $\chi$ is susceptibility, and $\theta$ is an angle between the position vector and $\mathrm{B}_{0}$ orientation. Since $d$ is a function of $\mathrm{B}_{0}$ orientation, the susceptibility-induced frequency shift is also dependent on the orientation of $\mathrm{B}_{0}{ }^{4,33}$

\subsection{Separation of chemical shift/exchange from susceptibility}

When $\mathrm{B}_{0}$ is applied to an object along the three orthogonal axes (i.e., $\mathrm{x}-, \mathrm{y}-$, or $\mathrm{z}$-axis), the three susceptibility-induced frequency shifts can be written as

$$
\begin{aligned}
& f_{s, x}(\vec{r})=d_{x}(\vec{r}) * \chi(\vec{r})=\frac{1}{4 \pi} \cdot \frac{3 \cos ^{2} \theta_{x}-1}{\vec{r}^{3}} * \chi(\vec{r}), \\
& f_{s, y}(\vec{r})=d_{y}(\vec{r}) * \chi(\vec{r})=\frac{1}{4 \pi} \cdot \frac{3 \cos ^{2} \theta_{y}-1}{\vec{r}^{3}} * \chi(\vec{r}), \\
& f_{s, z}(\vec{r})=d_{z}(\vec{r}) * \chi(\vec{r})=\frac{1}{4 \pi} \cdot \frac{3 \cos ^{2} \theta_{z}-1}{\vec{r}^{3}} * \chi(\vec{r}),
\end{aligned}
$$


where sub-index $\mathrm{x}, \mathrm{y}$, and $\mathrm{z}$ indicate the orientation of $\mathrm{B}_{0}$ and $\theta_{x}, \theta_{y}$, and $\theta_{z}$ represent the angle between the position vector $\vec{r}$ and the $\mathrm{B}_{0}$ field along the $\mathrm{x}, \mathrm{y}$, and $\mathrm{z}$-axes, respectively.

When we sum the three frequency shifts of the orthogonal orientations, it results in a null frequency shift:

$$
f_{s, x}(\vec{r})+f_{s, y}(\vec{r})+f_{s, z}(\vec{r})=\left\{\frac{1}{4 \pi} \cdot \frac{3\left(\cos ^{2} \theta_{x}+\cos ^{2} \theta_{y}+\cos ^{2} \theta_{z}\right)-3}{\vec{r}^{3}}\right\} * \chi(\vec{r})=0,
$$

because $\cos ^{2} \theta_{x}+\cos ^{2} \theta_{y}+\cos ^{2} \theta_{z}$ equals to 1 for the three orthogonal axes (see Supporting Information Section 1).

Using this property, we can measure the chemical shift/exchange-induced frequency shift by averaging the frequency shifts from the three orthogonal $\mathrm{B}_{0}$ orientations.

$$
\begin{aligned}
& f_{\text {average }}(\vec{r})=\frac{1}{3}\left\{f_{x}(\vec{r})+f_{y}(\vec{r})+f_{z}(\vec{r})\right\} \\
& =\frac{1}{3}\left[3 f_{c}(\vec{r})+\left\{f_{s, x}(\vec{r})+f_{s, y}(\vec{r})+f_{s, z}(\vec{r})\right\}\right]=f_{c}(\vec{r})
\end{aligned}
$$

Then, the susceptibility-induced frequency shift can be calculated by subtracting the chemical shift/exchange-induced frequency shift from the total frequency shift.

$$
f_{s}(\vec{r})=f(\vec{r})-f_{c}(\vec{r})
$$

From this susceptibility-induced frequency shift, one can measure the susceptibility by using a QSM algorithm.

This approach of separating the susceptibility and chemical shift/exchange requires data acquisition of three different $\mathrm{B}_{0}$ orientations that are orthogonal to each other. If an object has geometric symmetry, however, the number of scans can be reduced. For example, a cylindrical phantom has the geometric symmetry in the two short axes and, therefore, only two acquisitions, one with $\mathrm{B}_{0}$ along the long axis of the cylinder and the other with $\mathrm{B}_{0}$ along one of the short axes, are necessary. The missing frequency shift map can be generated by the $90^{\circ}$ rotation of the frequency shift map acquired with $\mathrm{B}_{0}$ along the short axis. Similarly, a spherical phantom can be scanned once to apply our method. The idea can be generalized for arbitrary $\mathrm{B}_{0}$ orientations using a least-squares minimization method (see Supporting Information Section 2). 


\section{METHODS}

\subsection{Numerical simulation}

A numerical simulation was designed to demonstrate that the summation of the three susceptibility-induced frequency shifts results in a null field (Eq. 5). Four numerical phantoms in the shape of heart, cylinder, sphere, and brain $^{34}$ were constructed in a $256^{3}$-voxel grid each. For the heart, cylinder, and sphere phantoms, the susceptibility of $0.1 \mathrm{ppm}$ was assigned. For the brain phantom, the following susceptibility values were assigned to subregions: caudate nucleus $=0.04$ ppm, putamen $=0.07 \mathrm{ppm}$, globus pallidus $=0.12 \mathrm{ppm}$, gray matter $=0.02 \mathrm{ppm}$, and white matter $=-0.02 \mathrm{ppm}$. The background had zero susceptibility. No chemical shift/exchange was assumed. Then, the susceptibility-induced frequency shifts of the three orthogonal $\mathrm{B}_{0}$ orientations were generated using a Fourier-based frequency shift calculation method. After that the three susceptibility-induced frequency shifts were summed to demonstrate that the result was a null field.

Another numerical simulation was performed to illustrate the process of the proposed method. A cylindrical phantom of the 30-voxel radius was embedded at the center of a $256 \times 256 \times 40$-voxel grid. The material inside the phantom was assumed to be fat with a chemical shift of $-3.5 \mathrm{ppm}^{35}$ and susceptibility of $0.65 \mathrm{ppm}^{28}$. The background was assumed to have zero susceptibility and chemical shift. A susceptibility-induced frequency shift map was calculated for each of the three orthogonal $\mathrm{B}_{0}$ orientations using the Fourier-based method. Then a chemical shiftinduced frequency shift map, which was confined to the cylinder with a $-3.5 \mathrm{ppm}$ frequency shift, was generated. The two maps were summed to yield a total frequency shift map for each $\mathrm{B}_{0}$ orientation. Our method of separating the susceptibility and chemical shift sources was applied to the three total frequency shift maps. As the first step, the three total frequency shift maps were averaged to produce a chemical shift-induced frequency shift map (Eq. 6). Then, this map was subtracted from each of the total frequency shift maps, providing the susceptibility-induced frequency shift maps (Eq. 7). From these frequency shift maps, susceptibility maps of each orientation were reconstructed by a QSM method ${ }^{36}$ with a regularization factor of 10 . For comparison, susceptibility maps were reconstructed from the total frequency shifts, which contained the chemical shift effect, using the same QSM method. 


\subsection{Phantom experiment}

Olive oil (O1514, Sigma-Aldrich, St.Louis, MO), bovine serum albumin (BSA) solution (100 mg/ml; A7906, Sigma-Aldrich), ferritin solution (0.43 mg/ml; F4503, Sigma-Aldrich), and iron oxide solution $\left(2.5 \cdot 10^{-3} \mathrm{mg} / \mathrm{ml}\right.$; PMC1N, Bang's Laboratories Inc., Fishers, IL) were used to test the proposed method. Each solution filled out two identical plastic cylinders (inner diameter $=28 \mathrm{~mm}$, outer diameter $=30 \mathrm{~mm}$, and height $=110 \mathrm{~mm}$ ). Then, the two cylinders were positioned in a large container such that one cylinder was parallel to $\mathrm{B}_{0}$ and the other was perpendicular to $\mathrm{B}_{0}$. The container was filled with distilled water. In a separate scan, a sphere phantom (diameter $=$ $40 \mathrm{~mm}$ ) filled with the olive oil was also constructed and tested.

All scans were conducted on a 3T MRI scanner (SIEMENS Tim Trio, Erlangen, Germany) with a 12-channel array coil. The scan started with a three-plane localizer. Then shimming was performed to reduce field inhomogeneity. For the main scan of the BSA, ferritin, and iron oxide solutions, data were acquired at room temperature using a 3D multi-echo gradient echo sequence with the following parameters: $\mathrm{FOV}=192 \times 192 \times 64 \mathrm{~mm}^{3}$, voxel size $=2 \times 2 \times 2 \mathrm{~mm}^{3}, \mathrm{TR}=64 \mathrm{~ms}$, $\mathrm{TE}=5$ to $23.75 \mathrm{~ms}$ with an echo spacing of $3.75 \mathrm{~ms}$, number of echoes $=6$, bandwidth $=330$ Hz/pixel, flip angle $=12^{\circ}$, GRAPPA factor $=2$, monopolar readout gradient, and total acquisition time $=2.03 \mathrm{~min}$. For the olive oil, the readout gradient was modified from monopolar to bipolar in order to achieve shorter echo spacing, because of the large chemical shift of the olive oil when compared to the others. The scan parameters were also modified to TR $=40 \mathrm{~ms}, \mathrm{TE}=1.5$ to 9.78 ms with an echo spacing of $0.92 \mathrm{~ms}$, number of echoes $=10$, bandwidth $=2000 \mathrm{~Hz} /$ pixel, and total acquisition time $=1.6 \mathrm{~min}$. To compensate for the artifacts in the bipolar gradient, the sequence was repeated with the opposite gradient polarity. ${ }^{37}$ After scanning each solution, the two cylinders (or a sphere) were replaced by cylinders (or a sphere) with distilled water. Then the scan was repeated to acquire a reference for a background field. ${ }^{38}$

For data processing, the k-space data of each solution were reconstructed to multi-echo complex images via GRAPPA reconstruction ${ }^{39}$ and multi-channel image combination ${ }^{40}$. For the olive oil data, the two same TE complex images of the opposite gradient polarity were averaged to generate a complex image. ${ }^{37}$ The background field was removed by dividing the complex image of the main scan by that of the reference. Then, a frequency shift map was derived using a linear least-squares fitting from the multi-echo data. For the cylindrical phantoms, the two frequency 
shift maps, one parallel and the other perpendicular to $\mathrm{B}_{0}$, were manually registered. After that, the third orientation frequency shift map was generated by a $90^{\circ}$ rotation of the frequency shift map whose cylinder was perpendicular to $\mathrm{B}_{0}$. The resulting map was registered to the other maps. For the spherical phantom, the frequency shift map was rotated by $90^{\circ}$ in two orthogonal orientations. Then the two rotated maps were registered to the original map. After that our method was applied to separate the susceptibility-induced frequency shift and chemical shift/exchange-induced

frequency shift. From the susceptibility-induced frequency shift maps, susceptibility maps were reconstructed using the QSM method with two different regularization factors (10 and 100). During the QSM reconstruction, voxels that contained the cylinder wall had low signal to noise ratios (SNR) and were excluded to avoid partial volume-induced errors. For comparison, susceptibility maps were also reconstructed from the total frequency shift maps. To quantify the results, a region of interest (ROI) was chosen at the central eight slices of the cylinder. Then the mean and standard deviation of the susceptibility and chemical shift/exchange were measured in the ROI.

All data processing was performed using MATLAB (MATLAB 2014b, MathWorks Inc., Natick, MA).

\section{RESULTS}

The simulation results of the four numerical phantoms in the shapes of the heart, cylinder, sphere, and brain confirm that the sum of the three orthogonal frequency shift maps generates a null frequency shift in all phantoms (Supporting Information Section 3), demonstrating the validity of Eq. 5 in Theory.

The process of separating the susceptibility and chemical shift/exchange is shown in Figure 1 for the numerical fat phantom. The total frequency shift maps of three orthogonal $\mathrm{B}_{0}$ orientations are illustrated in Figure 1a. When the three frequency shift maps are averaged to generate a chemical shift map (Fig. 1b), the frequency shift measured inside the cylinder is $-3.5 \mathrm{ppm}$, which is the same as the assigned chemical shift. This map is subtracted from each of the total frequency shift maps, generating the susceptibility-induced frequency shift maps, as shown in Figure 1c. When these maps are reconstructed for QSM, they result in consistent susceptibility estimations 
for all $\mathrm{B}_{0}$ orientations (Fig. 1e; left cylinder: $0.65 \pm 0.01 \mathrm{ppm}$, middle cylinder: $0.65 \pm 0.01 \mathrm{ppm}$, right cylinder: $0.65 \pm 0.00 \mathrm{ppm})$ that are equal to the assigned susceptibility value $(0.65 \mathrm{ppm})$ regardless of the $\mathrm{B}_{0}$ orientations. On the other hand, when susceptibility maps are reconstructed from the total frequency shifts in Figure 1a, the susceptibility values show significant variations (Fig. 1d; left cylinder: $4.16 \pm 0.60 \mathrm{ppm}$, middle cylinder: $4.16 \pm 0.60 \mathrm{ppm}$, right cylinder: -8.97 $\pm 0.01 \mathrm{ppm})$. These results demonstrate that the chemical shift/exchange-induced frequency shift produces susceptibility errors and streaking artifacts.

The experimental results using the cylindrical olive oil phantom are displayed in Figure 2. The same trend as in the numerical fat phantom is observed. The frequency shift maps from the three orthogonal $\mathrm{B}_{0}$ show orientation dependency (Fig. 2a). When the three maps are averaged, the chemical shift-induced frequency shift map reveals $-3.60 \pm 0.01 \mathrm{ppm}$ (Fig. 2b). This map shows no blooming artifacts, confirming that the chemical shift effect is localized to the source. After removing the chemical shift effect, the susceptibility-induced frequency shift maps (Fig. 2c) are reconstructed to generate the QSM maps (Fig. 2e). The results report consistent susceptibility for all orientations (left cylinder: $0.63 \pm 0.02 \mathrm{ppm}$, middle cylinder: $0.61 \pm 0.02 \mathrm{ppm}$, right cylinder: $0.63 \pm 0.01 \mathrm{ppm}$ ). The measurements show robustness to the regularization factors (Supporting Information, Table $\mathrm{S} 1$ ). When susceptibility maps are reconstructed from the total frequency shift maps that include the chemical shift effect, they result in large susceptibility variations (left cylinder: $11.4 \pm 0.8 \mathrm{ppm}$, middle cylinder: $10.6 \pm 1.1 \mathrm{ppm}$, right cylinder: $-10.2 \pm 0.2 \mathrm{ppm}$ ) and streaking artifacts (Fig. 2d). The results of the olive oil using a spherical phantom report the same trend and are summarized in Supporting Information Figure S2.

The susceptibility and chemical exchange measurements of the BSA, ferritin, and iron oxide solutions are listed in Table 1. In all solutions, the susceptibility measurements show more consistent results in the proposed method than in the conventional QSM reconstruction.

\section{DISCUSSION}

In this paper, a novel method that separates the susceptibility and chemical shift/exchange in a phantom without anisotropy susceptibility or microstructure is presented. The method yields 
quantitative maps of magnetic susceptibility and chemical shift/exchange and is tested in numerical simulations and phantom experiments.

When the susceptibility and chemical shift/exchange measurements from our method were compared to literature values, they showed a good correspondence. The measurements of the BSA solution (susceptibility: $-0.059 \pm 0.002 \mathrm{ppm}$, chemical exchange: $0.008 \pm 0.001 \mathrm{ppm}$ ) closely matched to the literature values (susceptibility: $-0.068 \mathrm{ppm}$, chemical exchange: $0.008 \mathrm{ppm}$; these values were scaled from Reference 29 for concentration and Lorentz sphere correction). The measurements of the olive oil (susceptibility: $0.62 \pm 0.01 \mathrm{ppm}$, chemical shift: $-3.60 \pm 0.01 \mathrm{ppm}$ ) were also well in agreement with the literature values (susceptibility: $0.65 \mathrm{ppm}$ and chemical shift: -3.46 ppm from vegetable oil measured in Reference 28; susceptibility: 0.75 ppm from oleic acid in Reference 41).

In previous studies, ${ }^{8-11}$ the quantification of chemical shift/exchange of a solution was performed using a reference chemical (e.g., dioxane), assuming no interaction between the reference and solution. In a more recent study, however, a non-negligible amount of interaction was reported ${ }^{30}$, undermining the results of previous studies ${ }^{8-11}$. In other studies, algorithms were proposed to separate susceptibility and non-susceptibility sources, assuming a piece-wise constant condition. ${ }^{27,28}$ Our approach, on the other hand, avoids potential complications from the reference chemical while allowing voxel-wise image reconstruction.

The proposed method requires three scans of orthogonal $B_{0}$ orientations when an object has an arbitrary shape. The number of scans can be reduced in an object with symmetry (e.g., sphere or cylinder). For example, a spherical phantom can be scanned just once. However, practical issues such as positioning the sphere, removing air bubbles, and performing the reference scan were difficult in the spherical phantom. As a result, it was used once to demonstrate the feasibility. Instead, cylindrical phantoms, which require two scans of orthogonal $\mathrm{B}_{0}$ orientations, are easier to handle. The two phantoms resulted in consistent measurements as demonstrated in Figure 2 and Supporting Information Figure S2.

When the alignment of the three orthogonally oriented dataset is not accurate, it may introduce errors in the estimations. This effect is analyzed in Supporting Information Section 6. The results suggest that the measurements are robust (less than $0.5 \%$ errors) for a small 
misalignment $\left(<5^{\circ}\right)$. Addtionally, the effects of the noise amplification of the proposed method is analyzed in Supporting Information Section 7.

In our study, a reference scan was acquired in order to eliminate the background field accurately. Alternatively, one may apply a background field removal method to reduce the scan time from the reference scan. ${ }^{42}$

Since the olive oil phantom has a large chemical shift-induced frequency shift (-3.58 ppm; $458.24 \mathrm{~Hz}$ in $3 \mathrm{~T})$, a shorter echo spacing time $(<1.09 \mathrm{~ms})$ was required for the GRE sequence to reconstruct the frequency shift maps from the phase maps. To reduce the echo spacing time, we modified the readout gradient of the sequence from monopolar to bipolar. Then, the two datasets with the opposite readout gradient were combined to avoid the phase modulations. ${ }^{37}$ This modified GRE sequence with the echo spacing of $0.92 \mathrm{~ms}$ allowed us to reconstruct the frequency shift maps of olive oil phantom without any artifacts.

In the phantom experiments, we experienced a $\mathrm{B}_{0}$ drift, which may induce measurement errors. ${ }^{43}$ To minimize the $\mathrm{B}_{0}$ drift-induced errors, we stabilized the $\mathrm{B}_{0}$ field through a sufficient amount of dummy scans before the main scan. The scan time was also reduced by using parallel imaging. ${ }^{39}$

Applying our method to in-vivo human brain is challenging because of the three orthogonal orientation scans required. Furthermore, the brain has additional sources of frequency shift, such as tissue compartmentalization ${ }^{13}$ and susceptibility anisotropy. ${ }^{6}$ These contributions have $\mathrm{B}_{0}$ orientation dependency and are not considered in our model. Additionally, a study has suggested that chemical exchange may have $\mathrm{B}_{0}$ orientation dependency in in-vivo. ${ }^{44}$ All of these will complicate our results when the method is applied directly to in-vivo. As a result, further improvement in methodology is necessary and this work will provide a stepping stone toward future research.

\section{CONCLUSION}

In this study, we developed a geometric approach for separating the susceptibility and chemical shift/exchange in a phantom without anisotropic susceptibility or microstructure. The 
proposed method yields accurate susceptibility and chemical shift/exchange measurements in the numerical simulation and phantom experiments. When susceptibility maps are reconstructed from the total frequency shift maps that include the chemical shift/exchange effect, they reveal large susceptibility variations and streaking artifacts depending on the $\mathrm{B}_{0}$ orientation. On the other hand, the reconstruction results from the susceptibility-induced frequency shift maps demonstrate correct susceptibility measurements with no artifacts. The proposed method is useful not only in measuring magnetic susceptibility and chemical shift/exchange but also in improving QSM reconstruction algorithms.

\section{ACKNOWLEDGEMENTS}

This work was supported by the National Research Foundation of Korea grant funded by the Korea government (MSIT; NRF-2018R1A2B3008445), Creative-Pioneering Researchers Program through Seoul National University, and Institute of Engineering Research at Seoul National University. 


\section{REFERENCE}

1. Abduljalil AM, Schmalbrock P, Novak V, Chakeres DW. Enhanced gray and white matter contrast of phase susceptibility-weighted images in ultra-high-field magnetic resonance imaging. J Magn Reson Imaging. 2003;18(3):284-290.

2. Duyn JH, van Gelderen P, Li TQ, de Zwart JA, Koretsky AP, Fukunaga M. High-field MRI of brain cortical substructure based on signal phase. Proc Natl Acad Sci USA. 2007;104(28):11796-11801.

3. Marques JP, Maddage R, Mlynarik V, Gruetter R. On the origin of the MR image phase contrast: an in vivo MR microscopy study of the rat brain at $14.1 \mathrm{~T}$. Neuroimage. 2009;46(2):345-352.

4. Schenck JF. The role of magnetic susceptibility in magnetic resonance imaging: MRI magnetic compatibility of the first and second kinds. Med Phys. 1996;23(6):815-850.

5. Haacke EM, Xu Y, Cheng YC, Reichenbach JR. Susceptibility weighted imaging (SWI). Magn Reson Med. 2004;52(3):612-618.

6. Lee J, Shmueli K, Fukunaga M, et al. Sensitivity of MRI resonance frequency to the orientation of brain tissue microstructure. Proc Natl Acad Sci U S A. 2010;107(11):51305135.

7. Haacke EM, Brown RW, Thompson MR, Venkatesan R. Magnetic resonance imaging: physical principles and sequence design. Vol 82: Wiley-Liss New York:; 1999.

8. Zhong K, Leupold J, von Elverfeldt D, Speck O. The molecular basis for gray and white matter contrast in phase imaging. Neuroimage. 2008;40(4):1561-1566.

9. Luo J, He X, d'Avignon DA, Ackerman JJ, Yablonskiy DA. Protein-induced water 1H MR frequency shifts: contributions from magnetic susceptibility and exchange effects. J Magn Reson. 2010;202(1):102-108.

10. Shmueli K, Dodd SJ, Li TQ, Duyn JH. The contribution of chemical exchange to MRI frequency shifts in brain tissue. Magn Reson Med. 2011;65(1):35-43.

11. Shmueli K, Dodd SJ, van Gelderen P, Duyn JH. Investigating lipids as a source of chemical exchange-induced MRI frequency shifts. NMR Biomed. 2017;30(4).

12. He X, Yablonskiy DA. Biophysical mechanisms of phase contrast in gradient echo MRI. Proc Natl Acad Sci U S A. 2009;106(32):13558-13563.

13. Wharton S, Bowtell R. Fiber orientation-dependent white matter contrast in gradient echo 
MRI. Proc Natl Acad Sci U S A. 2012;109(45):18559-18564.

14. Sati P, van Gelderen P, Silva AC, et al. Micro-compartment specific T2* relaxation in the brain. Neuroimage. 2013;77:268-278.

15. Shmueli K, de Zwart JA, van Gelderen P, Li TQ, Dodd SJ, Duyn JH. Magnetic Susceptibility Mapping of Brain Tissue In Vivo Using MRI Phase Data. Magn Reson Med. 2009;62(6):1510-1522.

16. Duyn J. MR susceptibility imaging. J Magn Reson. 2013;229:198-207.

17. Haacke EM, Liu S, Buch S, Zheng W, Wu D, Ye Y. Quantitative susceptibility mapping: current status and future directions. Magn Reson Imaging. 2015;33(1):1-25.

18. Wang Y, Liu T. Quantitative susceptibility mapping (QSM): Decoding MRI data for a tissue magnetic biomarker. Magn Reson Med. 2015;73(1):82-101.

19. Liu C, Li W, Tong KA, Yeom KW, Kuzminski S. Susceptibility-weighted imaging and quantitative susceptibility mapping in the brain. J Magn Reson Imaging. 2015;42(1):23-41.

20. Barbosa JHO, Santos AC, Tumas V, et al. Quantifying brain iron deposition in patients with Parkinson's disease using quantitative susceptibility mapping, R2 and R2. Magn Reson Imaging. 2015;33(5):559-565.

21. Chen W, Zhu W, Kovanlikaya I, et al. Intracranial calcifications and hemorrhages: characterization with quantitative susceptibility mapping. Radiology. 2014;270(2):496-505.

22. Langkammer C, Liu T, Khalil M, et al. Quantitative susceptibility mapping in multiple sclerosis. Radiology. 2013;267(2):551-559.

23. Deistung A, Schäfer A, Schweser F, Biedermann U, Turner R, Reichenbach JR. Toward in vivo histology: a comparison of quantitative susceptibility mapping (QSM) with magnitude-, phase-, and R2*-imaging at ultra-high magnetic field strength. Neuroimage. 2013;65:299-314.

24. Nam Y, Gho SM, Kim DH, Kim EY, Lee J. Imaging of nigrosome 1 in substantia nigra at 3T using multiecho susceptibility map-weighted imaging (SMWI). J Magn Reson Imaging. 2017;46(2):528-536.

25. Reeder SB, Pineda AR, Wen Z, et al. Iterative decomposition of water and fat with echo asymmetry and least-squares estimation (IDEAL): application with fast spin-echo imaging. Magn Reson Med. 2005;54(3):636-644.

26. Sharma SD, Hernando D, Horng DE, Reeder SB. Quantitative susceptibility mapping in 
the abdomen as an imaging biomarker of hepatic iron overload. Magn Reson Med. 2015;74(3):673-683.

27. Schweser F, Zivadinov R. Quantitative susceptibility mapping (QSM) with an extended physical model for MRI frequency contrast in the brain: a proof-of-concept of quantitative susceptibility and residual (QUASAR) mapping. NMR Biomed. 2018;31(12):e3999.

28. de Rochefort L, Brown R, Prince MR, Wang Y. Quantitative MR susceptibility mapping using piece-wise constant regularized inversion of the magnetic field. Magn Reson Med. 2008;60(4):1003-1009.

29. Leutritz T, Hilfert L, Busse U, Smalla KH, Speck O, Zhong K. Contribution of iron and protein contents from rat brain subcellular fractions to MR phase imaging. Magn Reson Med. 2017;77(5):2028-2039.

30. Leutritz T, Hilfert L, Smalla KH, Speck O, Zhong K. Accurate quantification of watermacromolecule exchange induced frequency shift: effects of reference substance. Magn Reson Med. 2013;69(1):263-268.

31. Marques JP, Bowtell R. Application of a fourier-based method for rapid calculation of field inhomogeneity due to spatial variation of magnetic susceptibility. Concepts Magn Reson B Magn Reson Eng. 2005;25(1):65-78.

32. Salomir R, de Senneville BD, Moonen CT. A fast calculation method for magnetic field inhomogeneity due to an arbitrary distribution of bulk susceptibility. Concepts Magn Reson B Magn Reson Eng. 2003;19B(1):26-34.

33. Chu SC, Xu Y, Balschi JA, Springer CS, Jr. Bulk magnetic susceptibility shifts in NMR studies of compartmentalized samples: use of paramagnetic reagents. Magn Reson Med. 1990;13(2):239-262.

34. Zubal IG, Harrell CR, Smith EO, Rattner Z, Gindi G, Hoffer PB. Computerized threedimensional segmented human anatomy. Med phys. 1994;21(2):299-302.

35. Bernstein MA, King KF, Zhou XJ. Handbook of MRI pulse sequences. Elsevier; 2004.

36. Liu J, Liu T, de Rochefort L, et al. Morphology enabled dipole inversion for quantitative susceptibility mapping using structural consistency between the magnitude image and the susceptibility map. Neuroimage. 2012;59(3):2560-2568.

37. Shin H-G, Oh S-H, Fukunaga M, et al. Advances in gradient echo myelin water imaging at 3T and 7T. NeuroImage. 2019;188:835-844. 
38. Lee J, van Gelderen P, Kuo LW, Merkle H, Silva AC, Duyn JH. T2*-based fiber orientation mapping. Neuroimage. 2011;57(1):225-234.

39. Griswold MA, Jakob PM, Heidemann RM, et al. Generalized autocalibrating partially parallel acquisitions (GRAPPA). Magn Reson Med. 2002;47(6):1202-1210.

40. Robinson S, Grabner G, Witoszynskyj S, Trattnig S. Combining phase images from multichannel RF coils using 3D phase offset maps derived from a dual-echo scan. Magn Reson Med. 2011;65(6):1638-1648.

41. Lide DR, editor. Handbook of chemistry and physics. 87th ed. NewYork: CRC Press; 2006-2007.

42. Schweser F, Robinson SD, de Rochefort L, Li W, Bredies K. An illustrated comparison of processing methods for phase MRI and QSM: removal of background field contributions from sources outside the region of interest. NMR Biomed. 2017;30(4):e3604.

43. Foerster BU, Tomasi D, Caparelli EC. Magnetic field shift due to mechanical vibration in functional magnetic resonance imaging. Magn Reson Med. 2005;54(5):1261-1267.

44. Pampel A, Muller DK, Anwander A, Marschner H, Moller HE. Orientation dependence of magnetization transfer parameters in human white matter. Neuroimage. 2015;114:136-146. 
(a)

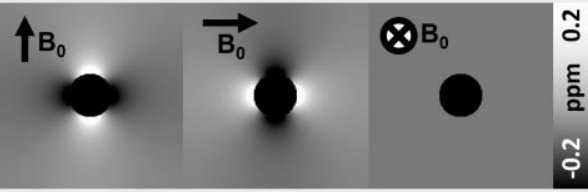

Total frequency shift maps

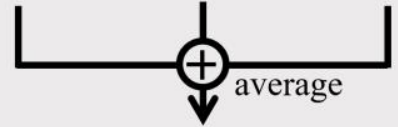

(b)

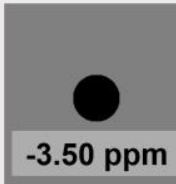

QSM

(d)

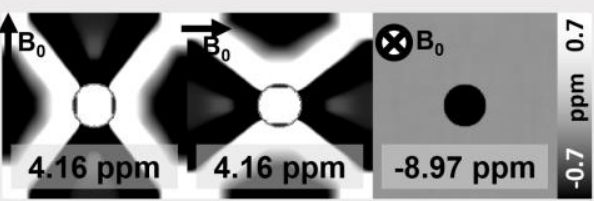

Susceptibility maps from total frequency shift maps

Chemical shift-induced frequency shift map (a) - (b) for each orientation

(c)

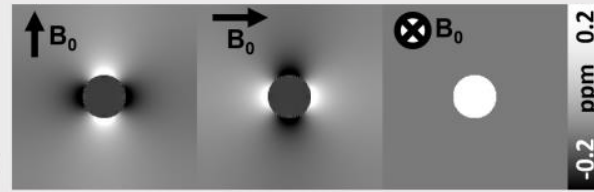

Susceptibility-induced frequency shift maps

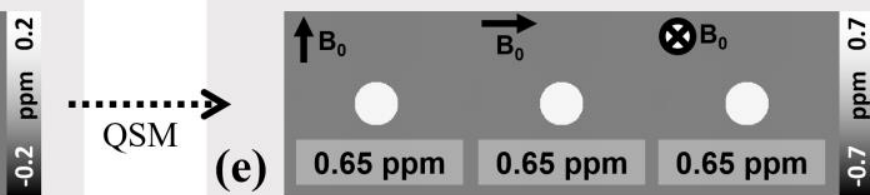

Susceptibility maps from susceptibility-induced frequency shift maps

Figure 1. Separation of the susceptibility and chemical shift in the numerical fat phantom. The three total frequency shift maps generated from the three orthogonal $\mathrm{B}_{0}$ orientations include the contributions of both susceptibility and chemical shift (a). The average of the three total frequency shift maps produces a chemical shift-induced frequency shift map (b). The susceptibility-induced frequency shift maps are generated by subtracting the chemical shift-induced frequency shift from the total frequency shift maps (c). The susceptibility maps reconstructed from the total frequency shift maps (d), and the susceptibility maps from the susceptibility-induced frequency shift maps (e) reveal significant differences with the latter reporting correct susceptibility estimations in all orientations. 
(a)

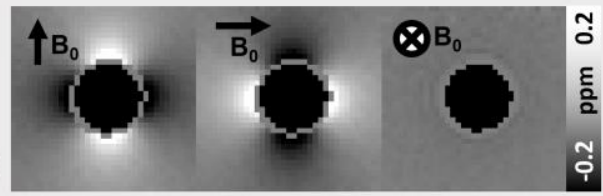

Total frequency shift maps

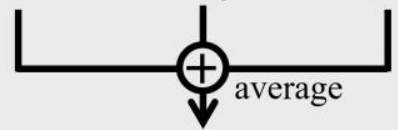

(b)

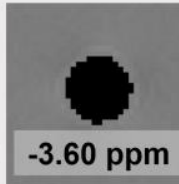

QSM

(d)

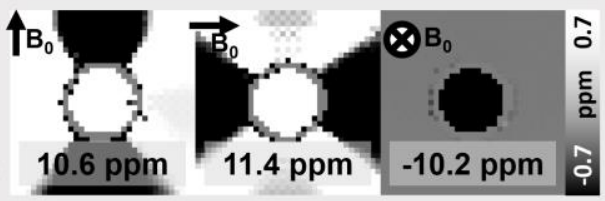

Susceptibility maps from total frequency shift maps

Chemical shift-induced frequency shift map (a) - (b) for each orientation

(c)

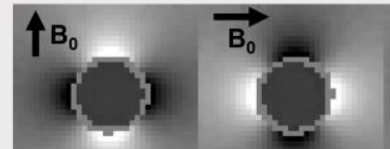

Susceptibility-induced frequency shift maps

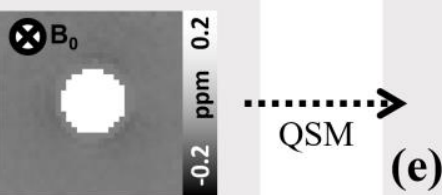

(e)

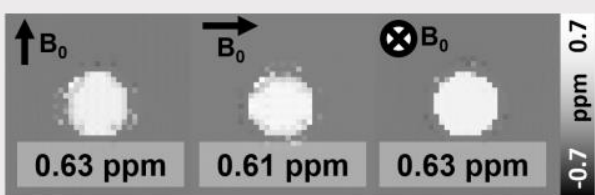

Susceptibility maps from susceptibility-induced frequency shift maps

Figure 2. Separation of the susceptibility and chemical shift in the cylindrical phantom with olive oil. The three total frequency shift maps generated from the three orthogonal Bo orientations contain the effect of both susceptibility and chemical shift (a). The average of the three total frequency shift maps produces the chemical shift-induced frequency shift map (b). The susceptibility-induced frequency shift maps are generated by subtracting the chemical shiftinduced frequency shift from the total frequency shift maps (c). The susceptibility maps reconstructed from the total frequency shift maps (d) and the susceptibility maps from the susceptibility-induced frequency shift maps (e) demonstrate significant differences with the latter reporting consistent and correct susceptibility measurements in all orientations. 


\section{Table 1}

Susceptibility $(\boldsymbol{\chi})$ and Chemical Shift/Exchange $(\boldsymbol{\Xi})$ Measurements for the Olive Oil, BSA, Ferritin, and Iron Oxide Solutions.

\begin{tabular}{|c|c|c|c|c|}
\hline & \multirow[b]{2}{*}{$\Xi, \mathbf{p p m}$} & \multicolumn{2}{|c|}{$\chi, \mathbf{p p m}$} & \multirow[b]{2}{*}{$\mathbf{B}_{0}$} \\
\hline & & $\begin{array}{c}\text { Susceptibility } \\
\text { measurements using } \\
\text { proposed method }\end{array}$ & $\begin{array}{c}\text { Susceptibility } \\
\text { measurements from } \\
\text { total frequency shift }\end{array}$ & \\
\hline \multirow{6}{*}{ Olive oil } & \multirow{3}{*}{$-3.60 \pm 0.01$} & $0.63 \pm 0.02$ & $11.4 \pm 0.8$ & (ब) \\
\hline & & $0.61 \pm 0.02$ & $10.6 \pm 1.1$ & $\theta$ \\
\hline & & $0.63 \pm 0.01$ & $-10.2 \pm 0.2$ & $0 \uparrow$ \\
\hline & \multirow{3}{*}{$-3.58 \pm 0.01$} & $0.66 \pm 0.01$ & $-5.07 \pm 0.63$ & $\otimes$ \\
\hline & & $0.66 \pm 0.01$ & $-4.02 \pm 1.08$ & $\Leftrightarrow$ \\
\hline & & $0.62 \pm 0.01$ & $-4.23 \pm 0.07$ & $\Leftrightarrow$ \\
\hline \multirow{3}{*}{ BSA } & \multirow{3}{*}{$0.008 \pm 0.001$} & $-0.057 \pm 0.001$ & $-0.082 \pm 0.001$ & (ब) \\
\hline & & $-0.058 \pm 0.002$ & $-0.080 \pm 0.002$ & $\theta$ \\
\hline & & $-0.061 \pm 0.001$ & $-0.038 \pm 0.001$ & \\
\hline \multirow{3}{*}{ Ferritin } & \multirow{3}{*}{$-0.005 \pm 0.001$} & $0.126 \pm 0.005$ & $0.137 \pm 0.006$ & (ब) \\
\hline & & $0.124 \pm 0.006$ & $0.136 \pm 0.008$ & $\theta$ \\
\hline & & $0.126 \pm 0.001$ & $0.109 \pm 0.001$ & \\
\hline \multirow{3}{*}{ Iron oxide } & \multirow{3}{*}{$-0.039 \pm 0.003$} & $0.28 \pm 0.01$ & $0.43 \pm 0.01$ & ब同 \\
\hline & & $0.31 \pm 0.01$ & $0.45 \pm 0.01$ & $\theta$ \\
\hline & & $0.32 \pm 0.01$ & $0.21 \pm 0.01$ & $\rho$ \\
\hline
\end{tabular}




\section{Supporting Information}

\section{Section 1. Three cosine terms in Equation 5 sums zero}

For the position vector $(\vec{r})$, the ratio of each component of the vector to the length of the vector can be expressed as the following cosine terms:

$$
\begin{aligned}
& \cos \theta_{x}=\frac{\|\mathrm{x}\|}{\|\vec{r}\|}, \\
& \cos \theta_{y}=\frac{\|\mathrm{y}\|}{\|\vec{r}\|}, \\
& \cos \theta_{z}=\frac{\|\mathrm{z}\|}{\|\vec{r}\|},
\end{aligned}
$$

where $\|\vec{r}\|^{2}=x^{2}+y^{2}+z^{2}$.

Therefore, $\cos ^{2} \theta_{x}+\cos ^{2} \theta_{y}+\cos ^{2} \theta_{z}=\frac{x^{2}+y^{2}+z^{2}}{\|\vec{r}\|^{2}}=1$, confirming Equation 5 to be zero. 


\section{Section 2. Least-squares solution for arbitrary $B_{0}$ orientation data}

In this section, we generalize our method for non-orthogonal $\mathrm{B}_{0}$ orientation data.

The Fourier transform of the total resonance frequency shift with $N$ different $\mathrm{B}_{0}$ orientations can be modeled as follows:

$$
\begin{aligned}
\Delta F_{i}(\vec{k}) & =F_{c}(\vec{k})+F_{s, i}(\vec{k})=F_{c}(\vec{k})+D_{i}(\vec{k}) X(\vec{k}), \\
D_{i}(\vec{k}) & =\frac{1}{3}-\frac{\left(k_{z} \cos \theta_{i}+k_{y} \sin \theta_{i} \cos \phi_{i}+k_{x} \sin \theta_{i} \sin \phi_{i}\right)^{2}}{k_{x}^{2}+k_{y}^{2}+k_{z}^{2}},
\end{aligned}
$$

where $\Delta F$ is the total frequency shift in k-space, $F_{S}$ is the susceptibility-induced frequency shift in k-space, $F_{C}$ is the chemical shift/exchange-induced frequency shift in k-space, $D$ is a dipole kernel in $\mathrm{k}$-space, and $X$ is susceptibility in $\mathrm{k}$-space. The sub-index $i$ represents a $\mathrm{B}_{0}$ orientation $(i=1,2$, $\ldots, N)$, and $\theta_{i}$ and $\phi_{i}$ are the angles of the $\mathrm{B}_{0}$ orientation in the spherical coordinate at the $i^{\text {th }}$ orientation.

Given a set of $N$ measurements, the frequency shifts can be reformulated as follows:

$$
\left[\begin{array}{c}
\Delta F_{1}(\vec{k}) \\
\Delta F_{2}(\vec{k}) \\
\vdots \\
\Delta F_{N}(\vec{k})
\end{array}\right]=\left[\begin{array}{cc}
1 & D_{1}(\vec{k}) \\
1 & D_{2}(\vec{k}) \\
\vdots \\
1 & D_{N}(\vec{k})
\end{array}\right]\left[\begin{array}{c}
F_{c}(\vec{k}) \\
X(\vec{k})
\end{array}\right]=D\left[\begin{array}{c}
F_{c}(\vec{k}) \\
X(\vec{k})
\end{array}\right] .
$$

If a sufficient number of data with different angles are acquired, the inverse problem in Equation S3 can be well-conditioned. Then, both chemical shift/exchange and susceptibility maps can be reconstructed using the least-squares estimation as shown below.

$$
\left[\begin{array}{c}
F_{c}(\vec{k}) \\
X(\vec{k})
\end{array}\right]=\left(D^{T} D\right)^{-1} D^{T}\left[\begin{array}{c}
\Delta F_{1}(\vec{k}) \\
\Delta F_{2}(\vec{k}) \\
\vdots \\
\Delta F_{N}(\vec{k})
\end{array}\right]
$$




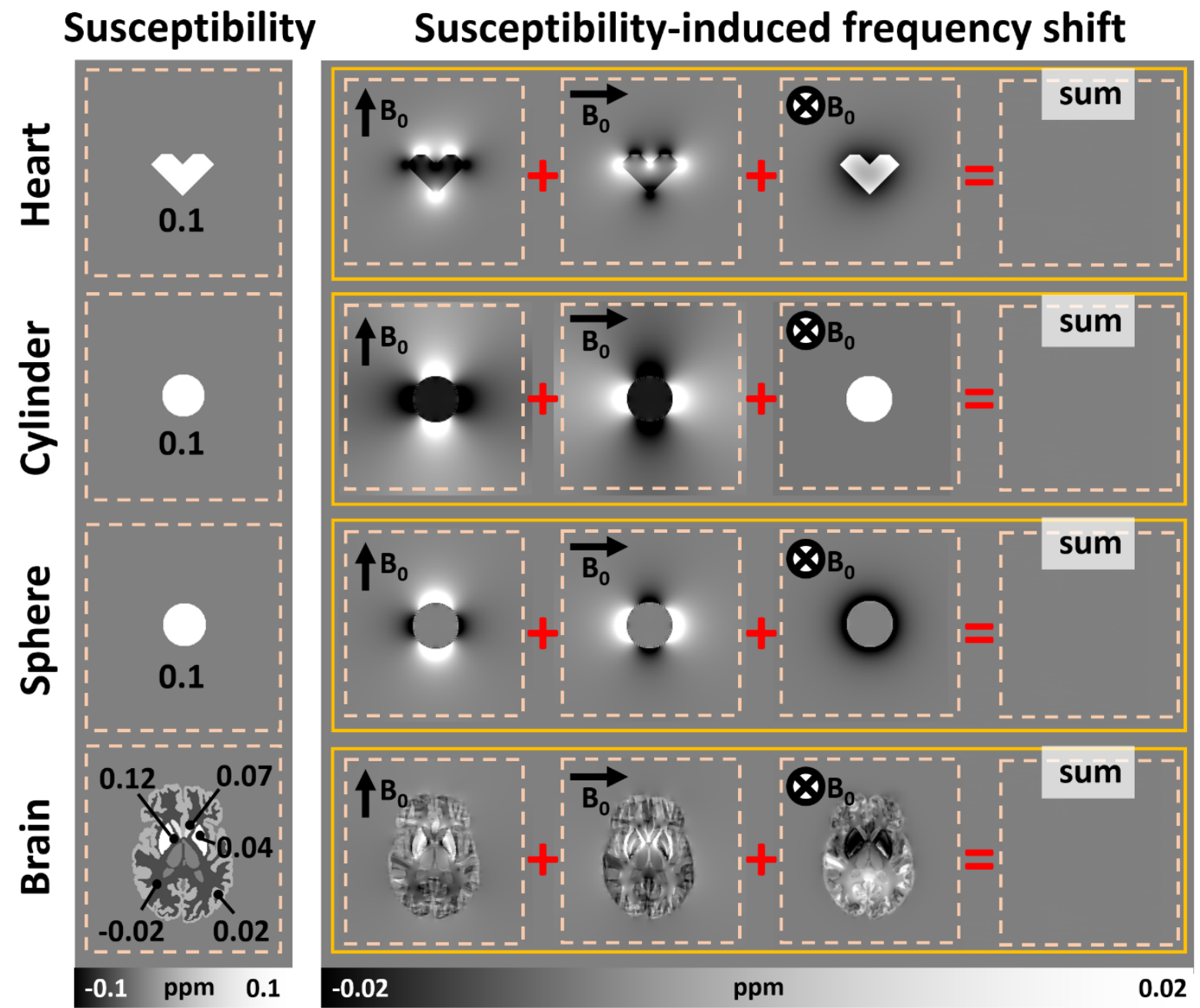

Figure S1. Susceptibility-induced frequency shifts in the heart-, cylinder-, sphere- and brainshaped numerical phantoms. The frequency shift maps demonstrate $\mathrm{B}_{0}$ orientation dependency. When the frequency shift maps of the three orthogonal $\mathrm{B}_{0}$ orientations are summed, a null field is generated. The susceptibility and frequency shifts are expressed in parts per million. 


\section{Section 4. Effects of two different regularization factors}

Table S1. Susceptibility measurements with two different regularization factors $(\lambda=10$ and $\lambda$

$=100)$.

\begin{tabular}{|c|c|c|c|}
\hline & \multirow{2}{*}{$\mathbf{B}_{\mathbf{0}}$} & \multicolumn{2}{|c|}{ Susceptibility measurements in ppm } \\
\hline & & $\lambda=10$ & $\lambda=100$ \\
\hline \multirow{3}{*}{ Olive oil } & ब & $0.63 \pm 0.02$ & $0.63 \pm 0.02$ \\
\hline & 母 & $0.61 \pm 0.02$ & $0.63 \pm 0.02$ \\
\hline & 甲† & $0.63 \pm 0.01$ & $0.63 \pm 0.01$ \\
\hline \multirow{3}{*}{ BSA } & ब2 & $-0.057 \pm 0.001$ & $-0.059 \pm 0.001$ \\
\hline & 母 & $-0.058 \pm 0.002$ & $-0.059 \pm 0.001$ \\
\hline & Ø1 & $-0.061 \pm 0.001$ & $-0.061 \pm 0.001$ \\
\hline \multirow{3}{*}{ Ferritin } & Q & $0.126 \pm 0.005$ & $0.130 \pm 0.004$ \\
\hline & 母 & $0.124 \pm 0.006$ & $0.131 \pm 0.004$ \\
\hline & Ø1 & $0.126 \pm 0.001$ & $0.126 \pm 0.001$ \\
\hline \multirow{3}{*}{ Iron oxide } & $\otimes$ & $0.28 \pm 0.01$ & $0.29 \pm 0.01$ \\
\hline & 8 & $0.31 \pm 0.01$ & $0.32 \pm 0.01$ \\
\hline & $\square \uparrow$ & $0.32 \pm 0.01$ & $0.32 \pm 0.01$ \\
\hline
\end{tabular}




\section{Section 5. Experimental results of the olive oil in the spherical phantom}

(a)

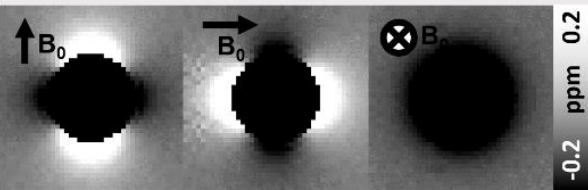

Total frequency shift maps

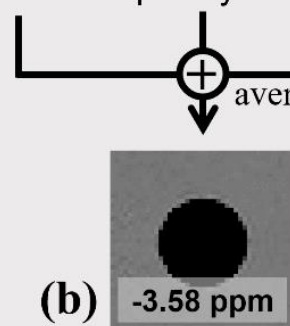

QSM

(d)

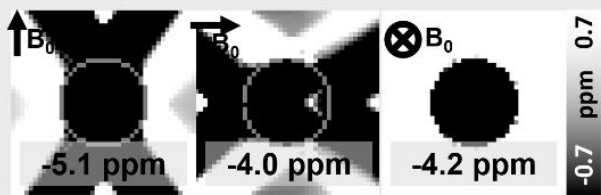

Susceptibility maps from total frequency shift maps

Chemical shift-induced frequency shift map

(c)

Susceptibility-induced frequency shift maps (a) - (b) for each orientation

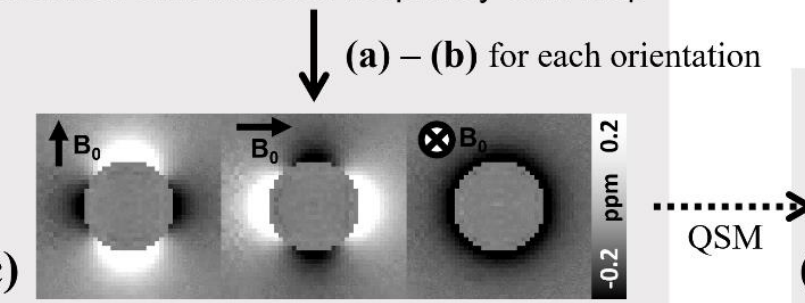

(e) $0.66 \mathrm{ppm} 0.66 \mathrm{ppm} \quad 0.62 \mathrm{ppm}$ 후

Susceptibility maps from susceptibility-induced frequency shift maps

Figure S2. Experimental results of the olive oil in the spherical phantom. The chemical shift (b, $3.58 \pm 0.01 \mathrm{ppm}$ ) and susceptibility (e, left sphere: $0.66 \pm 0.01 \mathrm{ppm}$, middle sphere: $0.66 \pm 0.01$ ppm, right sphere: $0.62 \pm 0.01 \mathrm{ppm}$ ) measurements are close to those of the olive oil in the cylindrical phantom. When the susceptibility maps are reconstructed from the total frequency shift maps with the chemical shift effect (b), significant susceptibility variations (left sphere: $-5.07 \pm$ $0.63 \mathrm{ppm}$, middle sphere: $-4.02 \pm 1.08 \mathrm{ppm}$, right sphere: $-4.23 \pm 0.07 \mathrm{ppm}$ ) and streaking artifacts are observed (d). As compared to the cylindrical phantom, the spherical phantom requires only one scan for the proposed method. However, the spherical phantom is not practical in some ways, and therefore, the cylindrical phantoms are used for the other solutions. 


\section{Section 6. Effects of misalignment}

Here, we design a numerical simulation to estimate the effects of misalignment of the cylinders in the experiment. A cylindrical phantom of the 30 -voxel radius is embedded at the center of a $256 \times 256 \times 256$-voxel grid. The material inside the phantom is assumed to have a chemical shift of $-3.5 \mathrm{ppm}$ and susceptibility of $0.65 \mathrm{ppm}$ with respect to the zero susceptibility and zero chemical shift of the background. One of the three cylinders is tilted by an angle $\theta$ centered at the middle grid point while the other two are perfectly aligned. Then, the three frequency shift maps are generated as described in the simulation. During the registration of the three frequency shift maps, the tilted angle was corrected because identifying and correcting for the misalignment in the acquired MR images is easy. Finally, the chemical shift and susceptibility maps are reconstructed using the proposed method. The percentage errors of the reconstructed values are calculated for a range of $\theta$ (from $0^{\circ}$ to $10^{\circ}$ ) for each axis.

The results are summarized in Figure S3. For both susceptibility and chemical shift, the misalignment effects are small, reporting less than $0.03 \%$ error for chemical shift and $0.5 \%$ for susceptibility when the misalignment error is less than $5^{\circ}$. The mean angular error in our experiment was $0.17 \pm 0.48^{\circ}$ with the maximum of $1.36^{\circ}$.
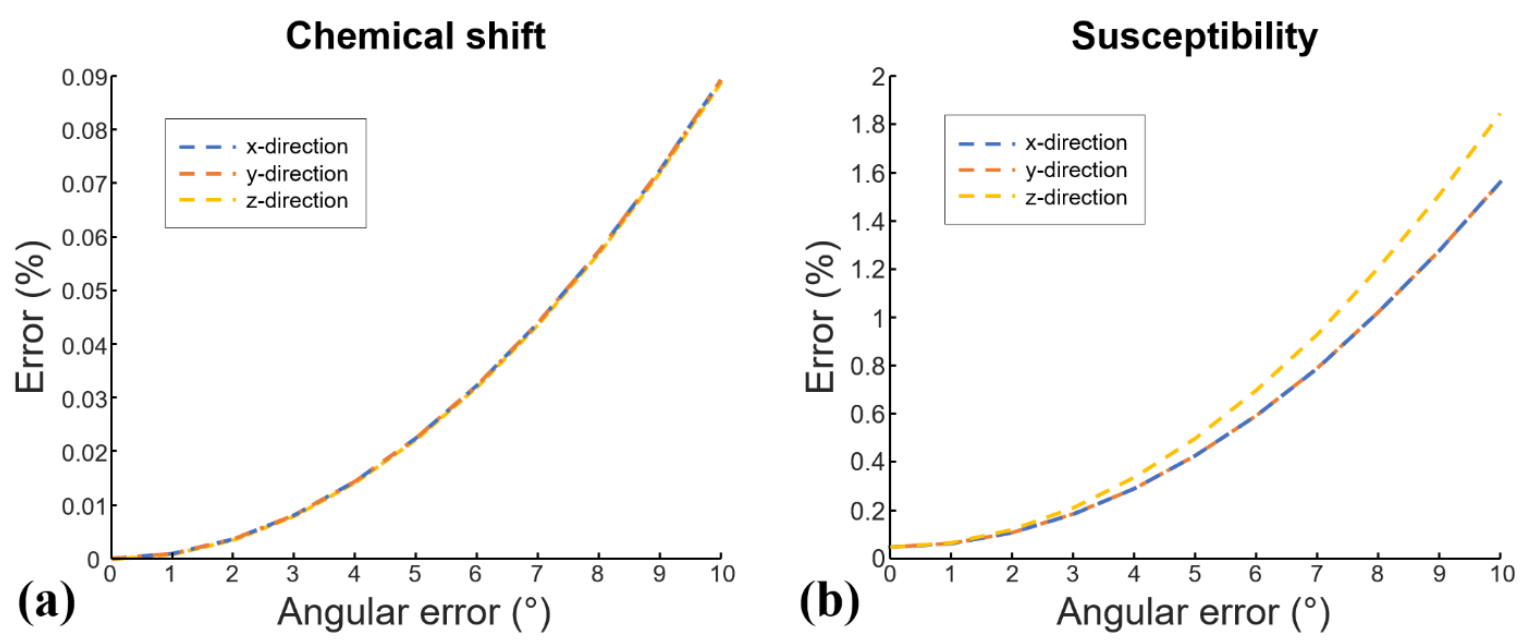

Figure S3. Evaluation of the misalignment effects of the cylindrical phantom. The plots of the percentage errors of the chemical shift/exchange (a) and susceptibility (b) measurements for the misalignment w.r.t. x- (blue), y- (orange), or z- (yellow) axis. The errors are small, suggesting the robustness of the method for the misalignment. 


\section{Section 7. Effects of noise amplification}

When considering noise $\left(n_{x}, n_{y}\right.$, and $n_{z}$ with a standard deviation of $\sigma$ and uncorrelated to each other), Equation 6 becomes:

$$
f_{c}(\vec{r})=\frac{1}{3}\left\{f_{x}(\vec{r})+n_{x}+f_{y}(\vec{r})+n_{y}+f_{z}(\vec{r})+n_{z}\right\}
$$

Hence, the noise component of the chemical shift/exchange map is $\frac{1}{3}\left(n_{x}+n_{y}+n_{z}\right)$ and the standard deviation is $\sqrt{E\left(\left(\frac{1}{3}\left(n_{x}+n_{y}+n_{z}\right)\right)^{2}\right)}=\frac{1}{\sqrt{3}} \sigma$. The final SNR of the chemical shift/exchange is increased by $\sqrt{3}$ due to the averaging. On the other hand, the noise effect in the susceptibility map is highly nonlinear and is dependent on the choice of a QSM reconstruction algorithm. 